\title{
End of life care for frail older people; do we have a consensus guideline on deprescribing?
}

\author{
Arjun Poudel ${ }^{1}$, Shakti Shrestha ${ }^{2}$, Anna Lukacisinova ${ }^{3}$, and Lisa Nissen ${ }^{4}$ \\ ${ }^{1}$ Queensland University of Technology \\ ${ }^{2}$ The University of Queensland \\ ${ }^{3}$ Charles University Faculty of Pharmacy in Hradec Kralove \\ ${ }^{4}$ Queensland University of Technology (QUT)
}

July 1, 2021

\begin{abstract}
Background: Deprescribing interventions have shown to improve medication appropriateness in older people. However, the evidence on the actual benefits and risks of deprescribing in older adults at the end of life are limited. Due to the lack of evidence on the safety and efficacy of medication in these populations, the most appropriate deprescribing approach is unclear. We aimed to conduct a narrative review of research on existing deprescribing guidelines targeted to frail older people at the end of life. Methods: A literature search was conducted in PubMed, Embase, CINAHL and Google Scholar to identify studies from inception to January 2021 on deprescribing guidelines/tools for frail older adults near end-of-life or palliative situation or life-limiting illnesses or limited life expectancy were included. Results: A total of nine studies were included. The deprescribing guidelines used in these studies were helpful to some extent in optimising medications in patients with limited life expectancy and life-limiting illnesses. Some of them have been tested in prospective studies that showed their usefulness in minimising the number of potentially inapproapriate medications. These studies however were not randomised and involved small sample sizes and had little insight into the clinical outcomes of using these tools. Conclusions: The existing tools and guidelines on deprescribing do not represent the end of life care nor address the medication appropriateness among individuals with a specific condition. An explicit and rigorous consensus-based guideline needs to be developed and tested in a well-designed clinical trial to measure clinically significant outcomes
\end{abstract}

\section{Hosted file}

Main text.doc available at https://authorea.com/users/422974/articles/528563-end-of-lifecare-for-frail-older-people-do-we-have-a-consensus-guideline-on-deprescribing

\section{Hosted file}

Table 1.docx available at https://authorea.com/users/422974/articles/528563-end-of-lifecare-for-frail-older-people-do-we-have-a-consensus-guideline-on-deprescribing 